\title{
Relationship between in vitro Motility of Pseudomonas plecoglossicida and Clinical Conditions in Affected Ayu
}

\author{
Se Chang Park ${ }^{1}$, Toshihiro Nakai ${ }^{1 *}$ and Akihiko Yuasa ${ }^{2}$ \\ ${ }^{1}$ Aquatic Pathobiology Laboratory, Graduate School of Biosphere Science, Hiroshima University, \\ Higashihiroshima 739-8528, Japan \\ ${ }^{2}$ Tokushima Prefectural Fisheries Institute, Hiwasa, Tokushima 779-2304 Japan
}

(Received May 21, 2002)

\begin{abstract}
This study was conducted to verify the previous contrasting results on the motility of Pseudomonas plecoglossicida and clinical conditions (bloody ascites) in affected ayu Plecoglossus altivelis. In the present field surveys at Tokushima Prefecture in 1999 and 2001, all $P$. plecoglossicida isolates from dead ayu, except one isolate from a fish with ascites, were non-motile irrespective of the presence of bloody ascites. When ayu were injected intramuscularly with nonmotile strains, either motile or non-motile isolates were obtained from the kidney of dead fish, while only motile ones were isolated after injection of motile strains. Bloody ascites manifested in most of the affected fish after injection of either type of the bacterium. In in vitro broth culture conditions, the non-motile strains altered into motile ones during two-week incubation. These results indicate that there are no relationship between in vitro motility of $P$. plecoglossicida and the presence of bloody ascites in affected ayu, and that the phenotypic alteration in the motility of $P$. plecoglossicida occurs under both in vitro and in vivo conditions.
\end{abstract}

Key words: Pseudomonas plecoglossicida, bacterial motility, bacterial hemorrhagic ascites, Plecoglossus altivelis, ayu

There have been two serious bacterial diseases among cultured population of ayu Plecoglossus altivelis in Japan since around 1990; cold water disease caused by Flavobacterium psychrophilum (Wakabayashi et al., 1994; lida and Mizokami, 1996) and bacterial hemorrhagic ascites caused by Pseudomonas plecoglossicida (Nakatsugawa and lida, 1996; Wakabayashi et al., 1996; Nishimori et al., 2000). Some antimicrobial agents such as florfenicol and sulfisozole are available to treat the former disease but not for the latter disease. Furthermore, as $P$. plecoglossicida infection emerges abruptly after such chemotherapy for cold water disease followed by heavy mortality, treatments for these two diseases must be considered inseparably.

$P$. plecoglossicida is homogeneous with respect to physiological, biochemical, and serological characteristics (Nakatsugawa and lida, 1996; Wakabayashi et al., 1996), and also consists of a single phage type (Park et al., 2000). However, in two previous papers the au- thors described contrasting results for the motility of the bacterium and the presence of bloody (hemorrhagic) ascites in affected fish; both of these characteristics were negative in one study (Nakatsugawa and lida, 1996), and both were positive in the other study (Wakabayashi et al., 1996). We reconfirmed that both motile and nonmotile types existed in strains of $P$. plecoglossicida which had been isolated from diseased ayu in geographically and chronologically different places (Park et al., 2000).

In order to solve this discrepancy, the relationship between the motility of $P$. plecoglossicida and different clinical conditions (bloody ascites) was investigated by isolation and characterization of bacteria from naturally infected fish with or without the clinical sign, and by in vitro and in vivo experiments using some selected motile and non-motile strains.

\footnotetext{
* Corresponding author

E-mail: nakaitt@hiroshima-u.ac.jp
} 


\section{Materials and Methods}

Field survey

Isolation of $P$. plecoglossicida from naturally affected ayu was performed at a private farm in Tokushima Prefecture in October 1999 (average fish weight: $56 \mathrm{~g}$ ) and February 2001 (3.6 g). Kidney tissues of fish were stamped on Trypto-Soya Agar (TSA, Nissui) and incubated at $25^{\circ} \mathrm{C}$ for 2 days. The isolates were identified by agglutination test with an anti-FPC951 rabbit serum. The motility of the isolates was examined directly by a wet mount method using a microscope.

\section{Bacterial strains}

Six strains of $P$. plecoglossicida were used for the in vivo and in vitro studies; three strains, FPC951 (=ATCC $700383^{\top}$ ), FPC941 (ATCC 700384), and PTH-9801 were motile, and the other three, AK-9510, PH-9501, and PTH-9802 were non-motile (Table 1). A lophotrichous flagellation was observed in motile strains, but not in non-motile strains. These bacteria were cultured on TSA at $25^{\circ} \mathrm{C}$ overnight prior to experiments. All in vitro experiments were carried out at $25^{\circ} \mathrm{C}$ unless cited otherwise.

\section{Infection of ayu with $\mathrm{P}$. plecoglossicida}

Groups of 20 ayu (average $4.2 \mathrm{~g}$ ) were injected intramuscularly (IM) with motile or non-motile strains at a dose of $10^{3} \mathrm{CFU} / \mathrm{fish}$ and then kept in $40 \mathrm{~L}$ plastic tanks with flow-through water at $20 \pm 1^{\circ} \mathrm{C}$. Mortalities and the presence of bloody ascites in dead fish were recorded daily for 2 weeks, and the kidneys of dead fish were subjected to bacterial isolation to confirm that death was due to $P$. plecoglossicida infection. Re-isolated bacteria from dead fish were tested for their motility by the wet mount method.

In vitro culture of $\mathrm{P}$. plecoglossicida under different conditions

Each motile or non-motile strain was incubated under different temperatures $\left(15,20,25\right.$ or $\left.30^{\circ} \mathrm{C}\right)$ on TSA, or in serially diluted TSB (Trypto-Soy Broth, Eiken) at $25^{\circ} \mathrm{C}$, or in TSB containing antimicrobial compounds (florfenicol $600 \mu \mathrm{g} / \mathrm{mL}$, chloramphenicol $500 \mu \mathrm{g} / \mathrm{mL}$, or sulfisozole sodium $2,000 \mu \mathrm{g} / \mathrm{mL}$ ) at $25^{\circ} \mathrm{C}$. $P$. plecoglossicida is highly resistant to these agents (Nakatsugawa and lida, 1996). Each culture on TSA was successively subcultured on a fresh medium everyday for 10 days. In broth cultures, a loopful of culture was transferred on TSA everyday and their one-day cultures at $25^{\circ} \mathrm{C}$ were tested for the motility. In a separate experiment, a non-motile PTH-9802 strain was inoculated into filter-sterilized $(0.45 \mu \mathrm{m}$ membrane filter) supernatants which had been collected from each 20 day-culture $\left(25^{\circ} \mathrm{C}\right)$ of $P$. plecoglossicida strains in TSB, incubated at $25^{\circ} \mathrm{C}$, and then the motility of PTH-9802 strain was examined everyday as described above. A similar experiment was carried out using these spent culture fluids that had been previously heated at $100^{\circ} \mathrm{C}$ for $3 \mathrm{~h}$.

\section{Results}

Clinical signs of fish and the motility of the isolates in field surveys

In the 1999 survey, bloody ascites was observed in 25 of 29 fish examined and almost all $P$. plecoglossicida isolates were non-motile (Table 2). Only one motile isolate was obtained from a dead fish with bloody ascites. There were no apparent clinical signs including bloody ascites in dead ayu in the 2001 survey and only non-motile $P$. plecoglossicida were isolated from the kidney of dead fish $(n=10)$.

Clinical signs of fish and the motility of $\mathrm{P}$. plecoglossicida in infection experiments

All of IM-injected fish with $P$. plecoglossicida strains, either motile or non-motile, were dead at 3rd to 5th day post-injection (Table 3). The characteristic bloody ascites was observed in the fish injected not only with the motile strains but also the non-motile strains at high incidence. Isolates from the fish injected with motile strains were all motile, while half of those from the fish injected with the non-motile strains were motile.

In vitro alteration in the motility of $\mathrm{P}$. plecoglossicida

No change in motility was observed during 10 days successive subcultures on TSA at the various incu-

Table 1. P. plecoglossicida strains in this study and their motility

\begin{tabular}{lllll}
\hline \multirow{2}{*}{ Strain } & \multicolumn{2}{c}{ Isolation from ayu } & \multirow{2}{*}{ Motility } & \multicolumn{1}{c}{ References } \\
\cline { 2 - 3 } & \multicolumn{1}{c}{ Location } & Year & & \\
\hline FPC951 $^{\text {a }}$ & Tokushima Prefecture & 1994 & + & Nishimori et al. 2000 \\
FPC941 $^{\text {b }}$ & Shiga Prefecture & 1994 & + & Nishimori et al. 2000 \\
PTH-9801 & Tokushima Prefecture & 1998 & + & Park et al. 2000 \\
AK-9510 & Kyoto Prefecture & 1995 & - & Nakatsugawa and lida 1996 \\
PH-9501 & Hiroshima Prefecture & 1995 & - & Nakatsugawa and lida 1996 \\
PTH-9802 & Tokushima Prefecture & 1998 & - & Park et al. 2000 \\
\hline
\end{tabular}

a Type strain, ATCC 700383

${ }^{\mathrm{b}}$ ATCC 700384 
Table 2. Clinical conditions of naturally affected ayu and motility of isolated $P$. plecoglossicida

\begin{tabular}{|c|c|c|c|c|c|}
\hline \multirow{2}{*}{ Year } & \multirow{2}{*}{$\begin{array}{c}\text { Average fish } \\
\text { weight }\end{array}$} & \multicolumn{2}{|c|}{ Bloody ascites in fish } & \multicolumn{2}{|c|}{ Motility of isolate } \\
\hline & & + & - & + & - \\
\hline 1999 & $56.0 \mathrm{~g}$ & 25 & 4 & 1 & 28 \\
\hline 2001 & $3.6 \mathrm{~g}$ & 0 & 10 & 0 & 10 \\
\hline
\end{tabular}

bation temperatures or in TSB at the different nutritional conditions or in TSB supplemented with three drugs. However, longer incubation (12 or 14 days) in drug-free TSB changed all three non-motile strains into motile ones, but no change was noticed in the motility of the motile strains during 20-day incubation (Table 4). No significant changes of $\mathrm{pH}$ values were noticed throughout the incubation period. Such alteration in the motility was observed within 8 days in the non-motile PTH-9802 strain when it was incubated with each 20-day-spent culture fluid (Table 5). This alteration was also observed in the spent culture fluids previously treated at $100^{\circ} \mathrm{C}$ for $3 \mathrm{~h}$ (data not shown). Once the non-motile strains turned to motile ones, the motility was retained throughout subsequent cultures on agar media.

\section{Discussion}

The present bacterial isolation from dead ayu in a culture facility in Tokushima Prefecture in 1999 and 2001 shows that there are no clear relationship between the presence of bloody ascites in diseased fish and in vitro motility of the causal organism; some of diseased fish were characterized by ascites but others were not, and

Table 3. Re-isolation of $P$. plecoglossicida from dead ayu after intramuscular injection with motile or non-motile strains

\begin{tabular}{llcccc}
\hline & & \multicolumn{4}{c}{ Number of fish } \\
\cline { 3 - 6 } $\begin{array}{c}\text { Injected strain } \\
\text { (dose : CFU/fish) }\end{array}$ & tested & dead $\begin{array}{c}\text { dead with } \\
\text { bloody } \\
\text { ascites }\end{array}$ & $\begin{array}{c}\text { from which motile } \\
P \text {. plecoglossicida } \\
\text { was isolated }\end{array}$ \\
\hline Motile & FPC951 $\left(1.6 \times 10^{3}\right)$ & 20 & 20 & 16 & 20 \\
strain & FPC941 $\left(1.8 \times 10^{3}\right)$ & 20 & 20 & 20 & 20 \\
& PTH-9801 $\left(2.0 \times 10^{3}\right)$ & 20 & 20 & 12 & 20 \\
\hline Non- & AK-9510 $\left(1.5 \times 10^{3}\right)$ & 20 & 20 & 16 & 10 \\
motile & PH-9501 $\left(3.4 \times 10^{3}\right)$ & 20 & 20 & 17 & 11 \\
strain & PTH-9802 $\left(1.2 \times 10^{3}\right)$ & 20 & 20 & 19 & 10 \\
\hline
\end{tabular}

Table 4. Alteration in the motility of motile and non-motile $P$. plecoglossicida strains in TSB

\begin{tabular}{ccccccccccccc}
\hline & & \multicolumn{7}{c}{ Strain } & \multicolumn{7}{c}{ Days after incubation in TSB at 25 $\mathrm{C}$} \\
\cline { 2 - 11 } & & 2 & 4 & 6 & 8 & 10 & 12 & 14 & 16 & 18 & 20 \\
\hline \multirow{2}{*}{ Motile } & FPC951 & $+^{\mathrm{a}}$ & + & + & + & + & + & + & + & + & + \\
strain & FPC941 & + & + & + & + & + & + & + & + & + & + \\
& PTH-9801 & + & + & + & + & + & + & + & + & + & + \\
\hline \multirow{2}{*}{ Non- } & AK-9510 & $-{ }^{\mathrm{b}}$ & - & - & - & - & - & + & + & + & + \\
motile strain & PH-9501 & - & - & - & - & - & - & + & + & + & + \\
& PTH-9802 & - & - & - & - & - & + & + & + & + & + \\
\hline
\end{tabular}

${ }^{a}$ motile

${ }^{\mathrm{b}}$ non-motile

Table 5. Alteration in the motility of a non-motile P. plecoglossicida strain (PTH-9802) in spent-culture fluids

\begin{tabular}{|c|c|c|c|c|c|c|c|c|c|c|c|}
\hline \multirow{2}{*}{\multicolumn{2}{|c|}{ Spent-culture fluids from }} & \multicolumn{10}{|c|}{ Days after incubation in spent-culture fluid at $25^{\circ} \mathrm{C}$} \\
\hline & & 1 & 2 & 3 & 4 & 5 & 6 & 7 & 8 & 9 & 10 \\
\hline \multirow{3}{*}{$\begin{array}{l}\text { Motile } \\
\text { strain }\end{array}$} & FPC951 & $-{ }^{a}$ & - & - & - & - & $t^{b}$ & + & + & + & + \\
\hline & FPC941 & - & - & - & - & + & + & + & + & + & + \\
\hline & PTH-9801 & - & - & - & - & - & + & + & + & + & + \\
\hline \multirow{3}{*}{$\begin{array}{l}\text { Non- } \\
\text { motile strain }\end{array}$} & AK-9510 & - & - & - & - & - & + & + & + & + & + \\
\hline & PH-9501 & - & - & - & - & - & - & - & + & + & + \\
\hline & РTH-9802 & - & - & - & - & + & + & + & + & + & + \\
\hline
\end{tabular}

\footnotetext{
${ }^{a}$ non-motile

${ }^{\mathrm{b}}$ motile
} 
almost all isolates were non-motile though only one motile isolate was obtained from a fish with ascites (Table 2). Infection experiments using selected motile and non-motile strains also clearly demonstrated that there are no essential relationship between the motility of the organism and manifestation of bloody ascites in fish (Table 3); ascites was produced in the fish injected with the non-motile strains and then the motile cells were isolated from some of these dead fish, but the motility of the motile strains was never lost after fish-passages and long-term subcultivation on agar plates. Therefore, it is concluded that the variations in the bacterial motility and the presence of bloody ascites were just reflected in the previous contrasting observations (Nakatsugawa and lida, 1996, Wakabayashi et al., 1996).

It is known that the synthesis of bacterial flagella is affected by culture conditions such as temperature or antibiotics (Hasegawa et al., 1982; Martinez and Gordee, 1966). In particular, we suspected that drugs such as florfenicol and sulfisozole frequently used for treatment of cold water disease may cause the variation in the motility of $P$. plecoglossicida. Contrary to our expectation, incubation of motile or non-motile strains of $P$. plecoglossicida with these drugs resulted in no changes in the motility during 10-day culture period, as well as the cultures at various temperatures or nutritional conditions. However, the prolonged cultivation in liquid medium induced alteration from non-motile to motile (Table 4). These results, together with field surveys and infection experiments described above, suggest that most of fresh isolates of $P$. plecoglossicida from naturally infected fish are non-motile and phenotypic alteration into motile one occurs during subsequent artificial manipulations. In the present study, unidentified heatstable substance(s) produced during bacterial growth is possibly associated with alteration in the motility of $P$. plecoglossicisa (Table 5). The precise mechanism remains for the future study.

\section{Acknowledgements}

The authors thank Dr. H. Wakabayashi for providing $P$. plecoglossicida strains and a rabbit antiserum. This work was supported in part by a Grant-in-Aid from the Ministry of Education, Science, Sports and Culture, Japan (No. 12660171), and the JSPS Research Fellowships for Young Scientists (S.C. Park).

\section{References}

Hasegawa, E., R. Kamiya and S. Asakura (1982): Thermal transition in helical forms of Salmonella flagella. J. Mol. Biol., 160, 609-621.

lida, Y. and A. Mizokami (1996): Outbreaks of coldwater disease in wild ayu and pale chub. Fish Pathol., 31, 157164.

Martinez, R. J. and E. Z. Gordee (1966): Formation of bacterial flagella. J. Bacteriol., 91. 870-875.

Nakatsugawa, T. and Y. lida (1996): Pseudomonas sp. isolated from diseased ayu, Plecoglossus altivelis. Fish Pathol., 31, 221-227. (in Japanese with English abstract)

Nishimori, E., K. Kita-Tsukamoto and H. Wakabayashi (2000): Pseudomonas plecoglossicida sp. nov., the causative agent of bacterial hemorrhagic ascites of ayu, Plecoglossus altivelis. Int. J. Syst. Evol. Microbiol., 50, 83-89.

Park S. C., I. Shimamura, M. Fukunaga, K-I. Mori and T. Nakai (2000): Isolation of bacteriophages specific to fish pathogen, Pseudomonas plecoglossicida, as a candidate for disease control. Appl. Environ. Microbiol., 66, 1416-1422.

Wakabayashi H., T. Toyama and T. lida (1994): A study on serotyping of Cytophaga psychrophila isolated from fishes in Japan. Fish Pathol., 29, 101-104.

Wakabayashi, H., K. Sawada, K. Ninomiya and E. Nishimori (1996): Bacterial hemorrhagic ascites of ayu caused by Pseudomonas sp. Fish Pathol., 31, 239-240. (in Japanese with English abstract) 